\title{
Design and manufacturing front hood for electric vehicle by carbon fibber
}

\author{
Paul Bere ${ }^{1}$, Calin Neamtu ${ }^{2, *}$, and Cristian Dudescu ${ }^{3}$ \\ ${ }^{1}$ Technical University of Cluj-Napoca, Manufacturing Engineering, Blvd. Muncii 103-105, Romania \\ 2 Technical University of Cluj-Napoca, Design Engineering and Robotics, Blvd. Muncii 103-105, \\ Romania \\ ${ }^{3}$ Technical University of Cluj-Napoca, Mechanical Engineering department, Blvd. Muncii 103-105, \\ Romania
}

\begin{abstract}
The polymeric reinforced composite materials (FRP) are a new materials group which have a lot of research reported in the last years. Our paper present the results obtained in case of use FRP for manufacturing an electric vehicle front hood in order for lightweight chassis and increase the autonomy. The design stage and 3D modelling of the mould, material design and manufacturing front hood from Carbon Fibber/Epoxy are presented.
\end{abstract}

\section{Introduction}

In the last time, the new materials as composite materials are thoroughly researched for a lot of researchers' team. They must find solutions to scientific challenges, and practical applications ever greater. These materials are used in high performance began, in areas such as aerospace, military or aviation. Step by step these advanced materials are used in our day by day life in different domain. Such materials are also fibre-reinforced polymer which has an accelerated development. FRP is composite materials made by plastic (polymer) matrix and reinforced fibres. These two components put together are very impressive mechanical properties in condition of low density.

Today FRP are in a continues interest and development and are intensive applied in automotive manufacturing industry, medicine, aircraft, motorsport, manufacturing machines industry or building construction. The McLaren Company used these materials in 1981 and built the first Formula One prototype car. Revolutionary car considered for that time, it brought a significant change in the car performance. In 2002, the first car made by carbon fibre polymer (CFRP) was produced.

The increasing demand on reduced emissions, lower fuel consumption and higher safety in the automotive industry requires not only a development of alternatively powered vehicles, but also consistent lightweight design. The use of lightweight materials such as glass or carbon FRP is a possible approach to achieve these goals. In contrast to metal materials FRP have very high values for specific stiffness and strength [1]. The usage of FRP structures within new vehicle concepts beneficially leads to a further enhancement of structural safety while lowering the vehicle's mass. The use of FRP for the Body-in-White (BIW) shows a

\footnotetext{
*Corresponding author: calin.neamtu@muri.utcluj.ro
} 
weight saving potential of up to $60 \%$ to $70 \%$ compared to metal materials [2-3]. This potential has been exploited for many years in motorsport and comes more and more into focus for conventional cars. In particular, for future alternatively powered vehicles, weight saving is directly linked to increased range which brings FRP-materials into such cars in greater extent (e.g. BMW i3 [4]).

In addition to the weight saving potential FRP also show a very high ratio of energy absorption per weight, compared to metal structures (CFK $100 \mathrm{~kJ} / \mathrm{kg}$ vs. $\mathrm{Al} \sim 50 \mathrm{~kJ} / \mathrm{kg}[5])$. This makes it a very interesting material for the crash-structures in a car. Actual applications can be found predominantly in sports cars but as soon as some shortcomings of those materials (cost, recycling, etc.) are solved, it is very likely that they are applied also in more vehicle types in the future. In [6-7] the authors presented an important application of FRP in a car door vehicle. Adaptive crash structures show potential for further increase of safety and lightweight performance. Different approaches with varying design goals and realizations have been discussed in recent publications. The main goals for the use of adaptive structures can be summarized as described in [8].

The numerical simulation of the FRP vehicles structures are a very important step in the design of components. [9-11]. In [12] the authors presented a methodology to design and manufacturing of the component from Formula One car. This can be applied in all the development CFRP car components.

In this paper, the authors presented the design and the manufacturing process for CFRP front hood in the electric car prototype case. The methodology for design of the component and the composite material layers' distribution were indicated. The vacuum assisted resin transfer mould (VRTM) to obtain the CFRP front hood are applied. The analysis of the typical load cases and computation of lateral, transversal and torsional stiffness of the front hood were done using finite element (FEA) commercial software.

\section{Design and manufacturing method}

\subsection{Design}

The front hood has been cut from general assembly shapes of an electric prototype vehicle. The design of this it was done using CATIA V5 software program using surface design and composite design modules. It was performed behind it based on the shape of the external surface of the model (Fig. 1. a, b). The hood are some fixing points and reinforcement zone for its hardening, this element was modelled in according with similar element from actual cars.

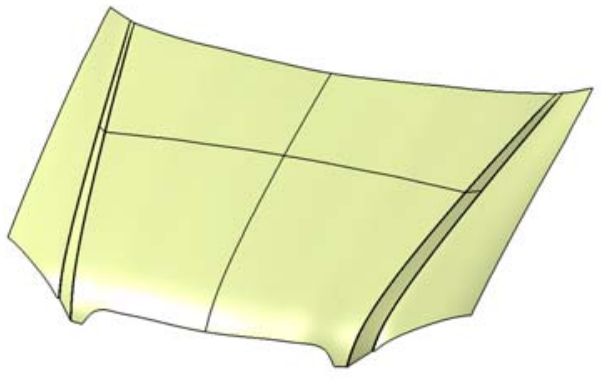

a.

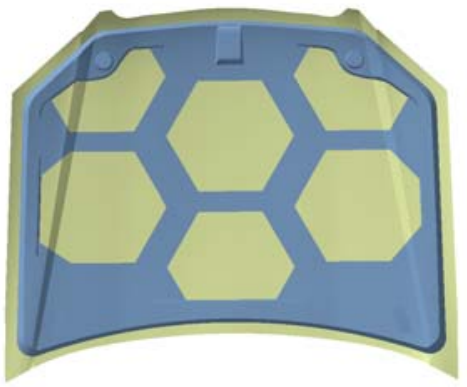

b.

Fig. 1 Front hood CAD prototype. 


\subsection{Numerical simulation}

The finite element model contains all geometrical components of the above described hood, the simulation was realized using ANSYS Workbench software [13] and the ACP tool for layup modelling of the composite materials. The composite material used in the simulation was defined as in the manufacturing process, the orthotropic material proprieties of the fabric epoxy carbon woven being those provided by the software material database (Epoxy Carbon Woven $(230 \mathrm{GPa}) \mathrm{Wet})$. The shell elements are used where the number of the elements was 21600. Connection between the inner and outer part is made by mesh connection in the contact areas. The bases for the analysis are the established static load cases [2-7] to determine the longitudinal, transversal and torsional stiffness. The hood is bended in cases of the lateral and transversal stiffness tests and distorted in the torsional stiffness test.

In all three tests, the hood is mounted in design position and constrained in the supporting points, the hinges in the rear hood area and the buffer points in the front area, respectively. The loads are applied in vertical direction and have for each load case the intensities shown in Figs. 2-4.

The assessment criterion is the total deflection of the load application point on the outer panel. Figs. 2-4 presents the total displacement distribution and its maximum value for investigated load cases. The obtained values give an indication about the hood stiffness in comparison with a similar one made from steel and ensure in this stage the proper composite and structural design. Comparison between experimental and numerical stiffness of the structure is necessary to be performed in the next step and consists future work.
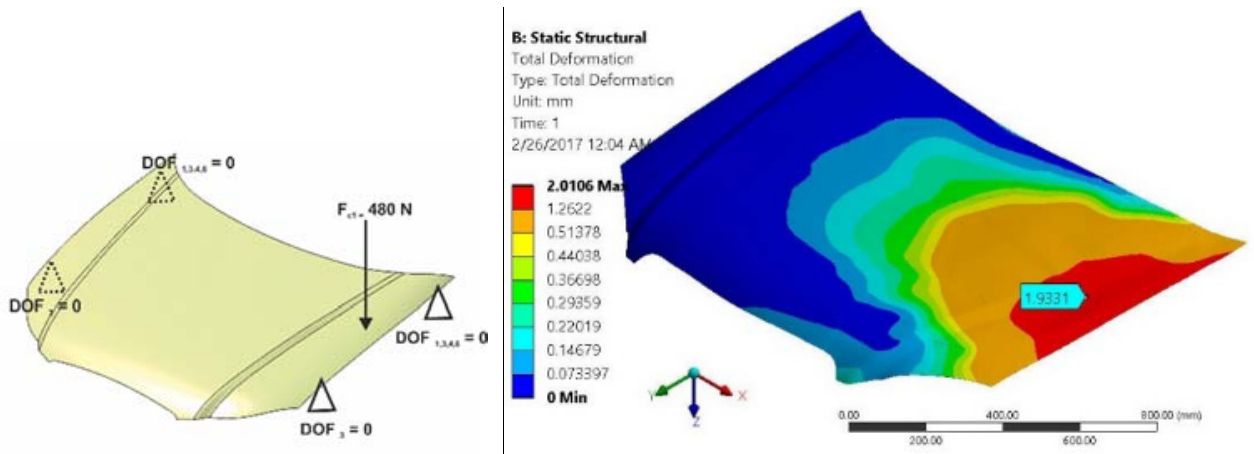

Fig. 2. Lateral stiffness of the composite hood: a) load case and b) total displacement.

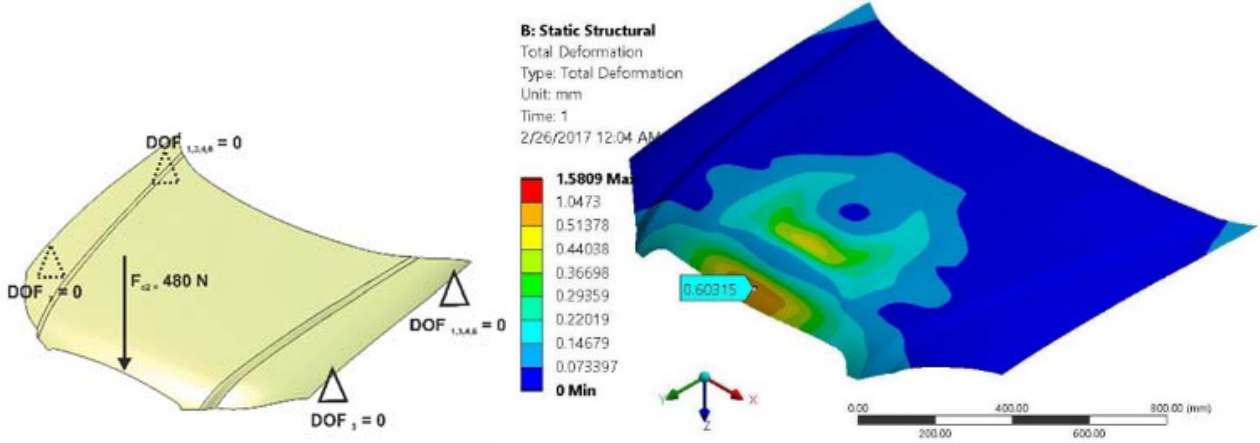

Fig. 3. Transversal stiffness of the composite hood: a) load case and b) total displacement. 

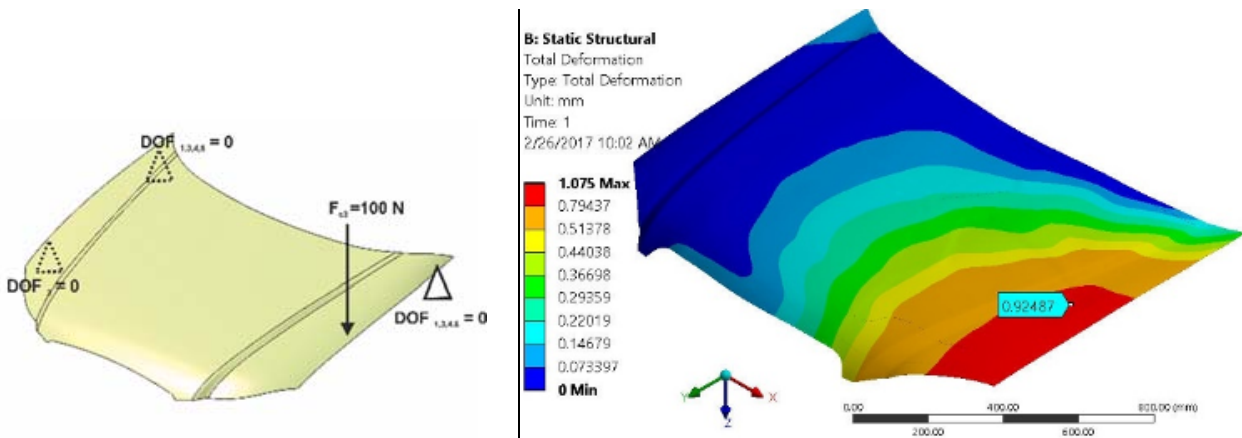

Fig. 4. Torsional stiffness of the composite hood: a) load case and b) total displacement.

\subsection{Materials and manufacturing method}

The materials used for manufacturing of front hood it was carbon fibre/epoxy. The balanced woven fabrics Twill 2 X2 by $200 \mathrm{~g} / \mathrm{m}^{2}$ were used. The distribution layers were $[90 / 0$ $\left.\pm 45_{2} / 90 / 0\right]$.

For the matrix L285 epoxy resin and hardener 286 from Lange-Ritter (Germany) was used. The mixing ratio according to the company prescription was 100:40 parts by weight.

The epoxy blocks were used to obtain the front hood mould. There was milling by CNC machines. To have a sufficient sealing surface of the vacuum bag the borders of the mould were extended by $100 \mathrm{~mm}$. Two moulds were manufacturing, one for exterior of the hood and one for back part (Fig. 5). The surface of the moulds was mechanical processing by glass paper sanding by 1000 grit.
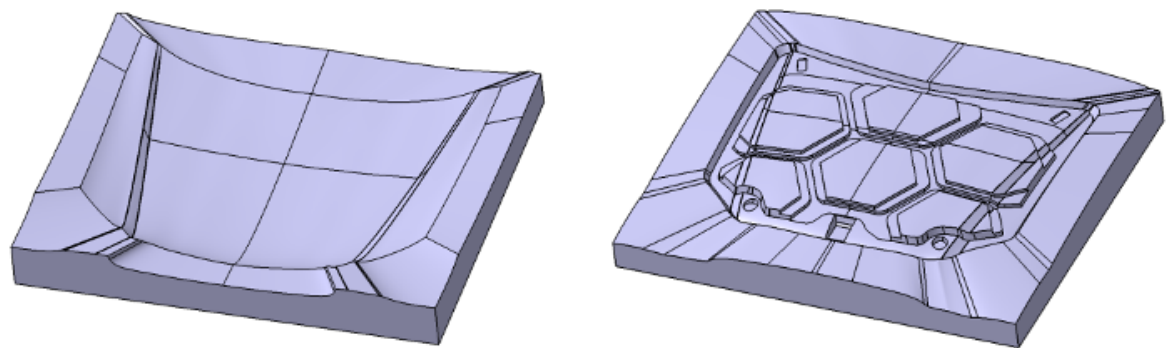

Fig. 5 3D model of the front hood moulds.

The surface was traded with several layers of Mould Sealer S31 agent supplied by the Jost Chemicals Company. This procedure closes the porosity of the mould's material. To realize a good thermal stability of the moulds a thermal treatment in an oven at $80^{\circ} \mathrm{C}$ for 8 hours' time were applied. To prevent the stick the CFRP laminating by the mould surface, several times of mould sealer tip Frekote B15 Aero by Loctite were applied.

The VRTM technology was applied to obtain the front hood by CFRP. The distribution of the carbon fibre layers was done according to the previous presented. The reinforced material was applied in the mould cavity. The CFRP reinforced material was covered by peel ply layer and breather fabric. On the border of the reinforced material the spiral tubing (wrapped in peel ply) were applied. All the system was covered by vacuum bag foil (Fig. 6). On the border of the mould the vacuum bag foil were seals by vacuum sealing type. The resin transfer was made on centrum of the mould and the vacuum valve was applied on the border. 
On the bag has been applied a vacuum pressure of -0.9 Bar 30 minutes tines. In this timer, the epoxy resin was mixing with hardener. To eliminate the air bubbles from mixing process the resin was vacuumed. After $30 \mathrm{~min}$., the epoxy resin was transferred in the carbon layers by five vacuum valves. The resin transfer was made progressive from a valve to another. The process was stopped when the resin covered all the composite materials and arrives on the border of the mould. The vacuum pressure eliminates all the resin excess in a trap filter.

Composite materials polymerization was done at $20^{\circ} \mathrm{C}$ for 12 hours. Supplementary thermal treatments of the composite were applied in the oven at $80^{\circ} \mathrm{C}$ for 8 hour. After complete polymerization, the composite pieces were released and the excess border materials were cut (Fig. 7). It was manufacturing two CFRP separate pieces. One for the top of the hood and one for back (reinforced structure). This two parts were bonded by a structural adhesive type Scotch Weld, EC-9323 B/A.

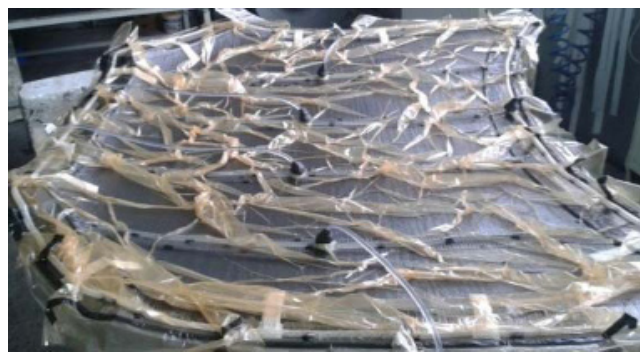

Fig. 6. Vacuum bag manufacturing method.

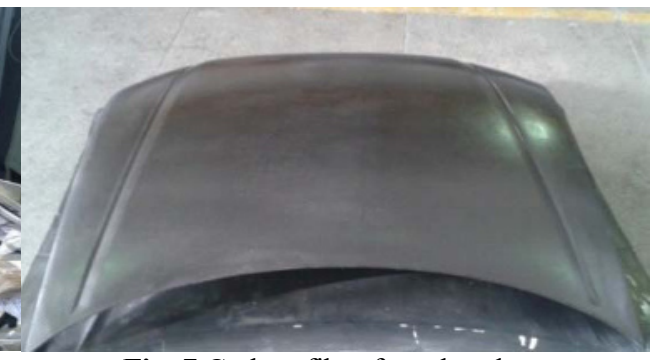

Fig. 7 Carbon fibre front hood.

\section{Results and discussions.}

A very good surface of the CFRP pieces on the mould part was obtained. This copies the mould surface and has a very good accuracy. On the back part of the carbon piece the surface is dimply typically for VARTM technology. The obtained degree of reinforcement was $64 \%$ in the weight fraction.

The design of the front hood was well done applied for CFRP components. The shape of the hood respected typical design for composites. The edges and ridges are adapted and the surfaces are connected by radius. No air bubbles or damage of reinforced materials are observed in a critical edge. The carbon fabric respected the initial disposal on the mould.

Comparatively with a similar hood designed of steel with metal sheets thickness of 0.5 $\mathrm{mm}$ the composite material hood has an increase in lateral stiffness of $52 \%$, in transversal stiffness of $8 \%$ and in torsional stiffness of $30 \%$ (simulation results). The density of CFRP material from front hood obtained is $1450 \mathrm{~kg} / \mathrm{m}^{3}$. The CFRP front hood presented in Fig. 4 has $3880 \mathrm{~g}$ weight. Comparative front steel hoods by $0.5 \mathrm{~mm}$ have $9400 \mathrm{~g}$ and from aluminium alloy by $0,8 \mathrm{~mm}$ have $8120 \mathrm{~g}$ weight. If we compare the weight of the hood realize form this metal pieces we obtained a weight decrease to $69 \%$ in a steel case and $53 \%$ in aluminium alloy case.

\section{Conclusions.}

The composite materials used to manufacture of the electric vehicle front hood have a significant weight reduction. This has a substantial contribution of the mass properties reduction of the electric vehicle and increases the autonomy of the car. The manufacturing technology to obtain the front hood is commonly used in high performance CFRP domain. 
The composite structure obtained is homogeneous well pressed, and the degree of reinforcement was $64 \%$. The exterior of the carbon/epoxy piece looks good without porosity. The weight reduction between steel front hood and presented one is 2,42 times and from aluminium alloy is 2,09 times lighter.

The FE analyses of the mechanical structure of the vehicle front hood indicates that the design of the composite material fulfil the requirements of the standard static tests.

This work was supported by a grant of the Romanian National Authority for Scientific Research and Innovation, CNCS/CCCDI-UEFISCDI, project number PN-III-P2-2.1-BG-2016-0210, within PNCDI III.

\section{References}

1. A. Ahmed, L. Wei, Thin-Walled Structures, 99 97-108 (2016).

2. M. Hamacher, R. Wohlecker, L. Ickert, Abaqus Users' Conference, May 19-22, Newport, Rhode Island, USA, 1-16 (2008).

3. C.E. Torricelli, L. Splendi and M. Pettazzoni, in Proceedings of the World Congress on Engineering WCE 2011, July 6 - 8, London, U.K (2011).

4. N. Bhaskar, P. Rayudu, International Journal of Current Engineering and Technology, 5 (2015).

5. S. M. Darwish, S. M. Elseufy, A. Ahmad, Journal of Management \& Engineering Integration, 762 (2014).

6. S. Darwish, H. M. A. Hussein, A. Gemeal, International Journal of Engineering \& Technology IJET-IJENS 12 (2012).

7. W. Tan, Y. Wang, L. Li, Y. Zhang, in Proceedings of the 2012 International Conference on Computer Application and System Modeling. Atlantis Press, (2012).

8. M. Grujicic, G. Arakere, V. Sellappan, J. C. Ziegert, F. Y. Koçer, D. Schmueser, Multidiscipline Modeling in Materials and Structures, 5 1-28 (2009).

9. Q. Liu, Y. Lin, Z. Zong, G. Sun, Q. Li, Composite Structures 97 231-238 (2013).

10. A. Zinno, E. Fusco, A. Prota, G. Manfredi, Composite Structures 92 2208-2219 (2010).

11. G. Gstrein, C. Kurzböck, J-M. Opelka, J. Krollmann, in Proceedings of 24th International Technical Conference on the Enhanced Safety of Vehicles, Göteborg, Sweden, 8-11 June, 1-12 (2015).

12. P. Bere, C. Neamtu, in Proceedings of 3rd International Conference on Quality and Innovation in Engineering and Management, Cluj-Napoca, Romania, 21-26 (2014).

13. ANSYS Workbench, Release 16.0, ANSYS, Inc., Canonsburg, USA (2015) 